\title{
IMPROVEMENT OF ENERGY CONSUMPTION IN SPECTRUM SENSING COGNITIVE RADIO NETWORKS USING AN EFFICIENT TWO STAGE SENSING METHOD
}

\author{
Hikmat N. Abdullah ${ }^{a}$, Hadeel S. Abed $^{b, *}$ \\ ${ }^{a}$ Department of Information and Communication Engineering, College of Information Engineering, AL-Nahrain \\ university \\ ${ }^{b}$ Department of Networks Engineering, College of Information Engineering, AL-Nahrain university \\ * corresponding author: hs_eng1992@yahoo.com
}

\begin{abstract}
Cognitive radio $(\mathrm{CR})$ is a wireless technology developed to improve the usage in the spectrum frequency. Energy consumption is considered as a big problem in this technology, especially during a spectrum sensing. In this paper, we propose an algorithm to improve the energy consumption during the spectrum sensing. The theoretical analysis to calculate the amount of energy consumption, using the proposed method during sensing stage as well as the transmission stage during transmitting a local decision to the fusion center FC, are derived. The proposed algorithm is using energy detection technique to detect the presence or absence of the primary user (PU). The proposed algorithm consists of two stages: the coarse sensing stage and fine sensing stage. In the coarse sensing stage, all the channels in the band are sensed shortly and the channel that have maximum (or minimum) energy is identified to make a dense fine sensing for confirming the presence of the PU signal (or hole). The performance of the proposed algorithm is evaluated in two scenarios: non-cooperative, and cooperative in both the AWGN and Rayleigh fading channels. The simulation results show that the proposed method improves the energy consumption by about $40 \%$ at a low SNR values, when compared with the traditional methods based on a single sensing stage and more advanced method based on censoring and sequential censoring algorithms.
\end{abstract}

KEYWORDS: cognitive radio; spectrum sensing; energy detection; energy consumption; cooperative sensing; non-cooperative sensing; coarse-fine sensing.

\section{INTRODUCTION}

The concept of a cognitive radio is that unlicensed users (secondary users) can use the spectrum owned by licensed users (primary users), while they shouldn't make an interference to the primary users when they are using the spectrum. Thus, to achieve the technique of a cognitive radio, a secondary user must have the ability to measure, sense and learn the channel characteristics and availabilities [1]. One of the main challenges in the CR networks is the high energy consumption, especially in battery-powered terminals. In order to identify the unused spectrum portions, the cognitive users (CUs), are enforced to sense it for a specific period, this process causes an excessive energy consumption [2]. Different spectrum sensing techniques are available, such as energy detection, matched filter detection, waveform-based sensing, and cyclostationarity-based sensing. Energy detection is a simple technique for spectrum sensing and is one of the most commonly employed spectrum sensing schemes. It does not require any prior knowledge about the primary users' (PUs) signals [3]. Two scenarios of spectrum sensing are usually used, a non-cooperative scenario and cooperative scenario (CSS). In the noncooperative scenario, a single user is used to make the sensing, while in the cooperative scenario, mul- tiple cognitive users sense in cooperation to reduce the effect of fading [3]. In CSS, during the transmission stage, the local sensing results are reported to a central entity, called the fusion centre (FC). The $\mathrm{FC}$ is in charge of making a global decision regarding the spectrum occupancy by applying a specific fusion rule (FR) 2]. There are two popular schemes used to represent the local result: a hard-based scheme and a soft based scheme. In the hard scheme, all the CUs convey their local decisions as a single bit per a $\mathrm{CU}$ towards the FC consecutively: bit "1" indicates the $\mathrm{PU}$ presence and bit "0" indicates the PU absence. In soft scheme, the sensing result is usually quantized by a large number of bits 4 . In this work, the hard scheme is used, because it consumes less energy. The rule that is employed by the $\mathrm{FC}$ is the $\mathrm{OR}$ rule, which implies that if at least one CU makes a local decision of busy (or 1), the global decision will be busy (or 1). Otherwise; the global decision will be free (or 0) 4].

Many works have proposed the methods to reduce the energy consumption. Sequential sensing, as an algorithm to reduce the average number of sensors required to reach a decision, is thoroughly studied in [5-8]. A truncated sequential sensing technique is employed in 9 to reduce the sensing time of the CR network, where many thresholds are used. In [10, the CUs are divided into non-disjoint subsets in a 
way that only one subset senses the spectrum, while the other subsets stay in a low power mode. In [11, a censoring and combination of censoring and truncated sequential sensing are presented to reduce the energy consumption. However, although these approaches improve the energy consumption, they have some drawbacks. In censoring approach, two thresholds are used, this increases the complexity and due to the whole spectrum being sensed, it also increases the energy consumption. The method of a combination of censoring and truncation have a weakness in the performance of detection, since it stops the sensing process once the accumulated energy crosses the threshold without sensing other channels, many of which have higher accumulated energy i.e. more probable existence of the PU signal. In [12, the coarse sensing approach is used to find the available channel in a combination with the sequential sensing scheme. Although this method reduces the sensing time, it also has a problem that it uses sequential sensing, which does not select the optimal channel available since the sensing is stopped quickly when the channel available is found without checking other channels. Furthermore, for low values of the SNR, this method consumes high energy, because it cannot produce a local decision early and continue to take more samples. The fading effect is also not taken into consideration in this method. In this paper, we proposed a sensing method that overcomes the problems mentioned in the previous works [11, 12]. It makes sensing in two stages: the first stage is to shortly sense all the channels in the spectrum with a low number of samples and then identify the channel that have the maximum accumulated energy (the channel that is more likely to have a PU signal). Then, a fine sensing is done only on this channel with higher number of samples for verification. The rest of the paper is organized as follows: in Section 2, the spectrum sensing concept using energy detection is explained. Section 3 presents the proposed method with corresponding theoretical analysis. Section 4 gives the simulation results with a necessary discussion, while Section 5 outlines the conclusions deduced.

\section{ENERGy DETECTION TECHNIQUE}

Energy detection is one of the widely used spectrum sensing techniques since it has a low complexity and it does not need any knowledge about PUs signals. In the CR networks, the CR checks the spectrum allocated to the PU and when it detects the absence of the $\mathrm{PU}$ transmission, it starts the data transmission to its receiver. The received samples at the $\mathrm{CU}$ receiver are [3]:

$$
Y(n)=h_{\mathrm{ps}} \theta X_{\mathrm{p}}(n)+W(n),
$$

where $X_{\mathrm{p}}(n)$ is the signal of the $\mathrm{PU}, h_{\mathrm{ps}}$ is the channel gain between the $\mathrm{PU}$ and the $\mathrm{SU}, W(n)$ is the Additive White Gaussian Noise (AWGN) at the SU receiver, and $\theta$ is the PU activity indicator, which can take one out of two values:

$$
\theta= \begin{cases}0 & \text { for } H_{0} \text { hypothesis } \\ 1 & \text { for } H_{1} \text { hypothesis }\end{cases}
$$

when the PU is active, it is referred to as hypothesis $H_{1}$, while in the case of inactivity, the PU is referred to as hypothesis $H_{0}$. The false alarm and detection probabilities are evaluated by comparing the detector decision metric with a pre-set threshold $\lambda$. The decision metric (DMED) is defined by the energy of the captured samples during the observation window $t$ :

$$
\mathrm{DMED}=\frac{1}{N} \sum_{n=1}^{N}|Y(n)|^{2},
$$

where $N=t F_{\mathrm{S}}$ is the number of sensing samples and $F_{\mathrm{s}}$ is the sampling frequency. The probabilities of false alarm and detection are evaluated by:

$$
\begin{aligned}
& P_{\mathrm{f}}=\operatorname{Pr}\left(\mathrm{DMED}>\lambda \mid H_{0}\right), \\
& P_{\mathrm{d}}=\operatorname{Pr}\left(\mathrm{DMED}>\lambda \mid H_{1}\right) .
\end{aligned}
$$

\section{The PROPOSED METHOD - IMPROVED COARSE-FINE SENSING METHOD)}

The energy consumption during the spectrum sensing process consumes energy in both sensing and transmission stages. The proposed algorithm achieves energy saving in the sensing stage, and it also improves the performance detection. Figure 1 shows the flowchart of the design procedure of the proposed algorithm. This algorithm consists of two stages: a coarse sensing stage and fine sensing stage. In coarse stage, the CU senses a fixed spectrum length and a fixed number of channels with fewer number of sensed samples (e.g. $16 \%$ of the number of samples used in a typical sensing process). The accumulated energies obtained from all channels during the coarse sensing are collected and then a fine sensing is applied to the channel that has the maximum accumulated energy. Fine sensing senses all samples in the channel selected to the confirmation. In order to ensure that this signal is a PU signal (not noise), the accumulated energy in the channel is compared with a threshold $\lambda$ to produce a final decision about the PU signal activity.

If we want to detect a hole in the spectrum, the same procedure is repeated, but fine sensing is applied to the channel that has minimum accumulated energy. In general, the amount of the energy consumption achieved by the $j$ th cognitive user $C_{j}$ is given by

$$
C_{j}=C_{\mathrm{s}, j}+C_{\mathrm{t}, j}
$$

with

$$
C_{\mathrm{s}, j}=N C_{\mathrm{ss}, j},
$$

where $C_{\mathrm{s}, j}$ and $C_{\mathrm{t}, j}$ are the energies consumed by the $j$ th $\mathrm{CU}$ in sensing and transmission (per bit) stages 


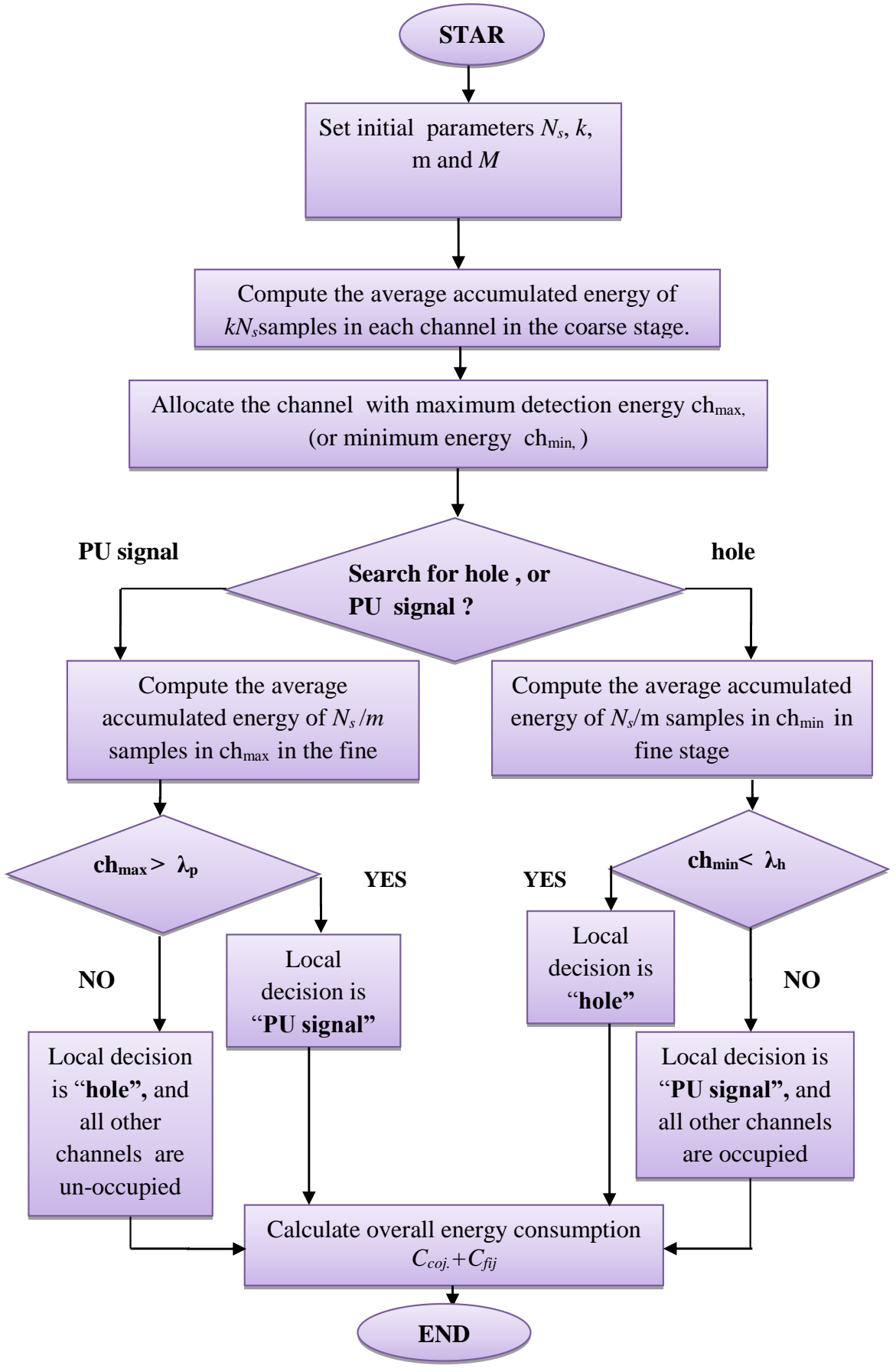

Figure 1. Flow chart of the proposed sensing method.

respectively, $N$ is the number of sensed samples, and $C_{\mathrm{ss}, j}$ is the energy consumption per sample.

As stated in [11, 12, $C_{\mathrm{ss}, j}$ is fixed and depends only on the sampling rate and the energy consumption of the sensing module, it is computed assuming $P_{\mathrm{d}}=$ 1 (i.e., $\left.C_{\mathrm{ss}, j \mid P_{\mathrm{d}}=1}\right)$.. When $P_{\mathrm{d}}$ is decreased, $C_{\mathrm{ss}, j}$ is increased since the energy detector will produce false decision, which leads to a repetition of the sensing process. According to this discussion, $C_{\mathrm{ss}, j}$ will be written as 13, 14]

$$
C_{\mathrm{ss}, j}=C_{\mathrm{ss}, j \mid P_{\mathrm{d}}=1}+C_{\mathrm{ss}, j \mid P_{\mathrm{d}}=1}\left(1-P_{\mathrm{d}}\right) .
$$

Considering a sensor used by the $\mathrm{CU}$ based on the
IEEE 802.15.4/ZigBee radios. The sensing energy for each decision consists of two parts: the energy consumption involved in listening over the channel and in making the decision and the energy consumption of the signal processing part for modulation, signal shaping,..etc. The number of samples per detection interval used in our simulation is chosen to be 5 , according to [15]. This interval corresponds to a detection time of $1 \mu \mathrm{s}$. Considering the fact that the typical circuit power consumption of ZigBee is approximately $40 \mathrm{~mW}$, the energy consumed for listening is approximately $40 \mathrm{~nJ}$. Therefore, we conclude that the energy consumption per sample is $40 \mathrm{~nJ} / 5=8 \mathrm{~nJ}$. So, 

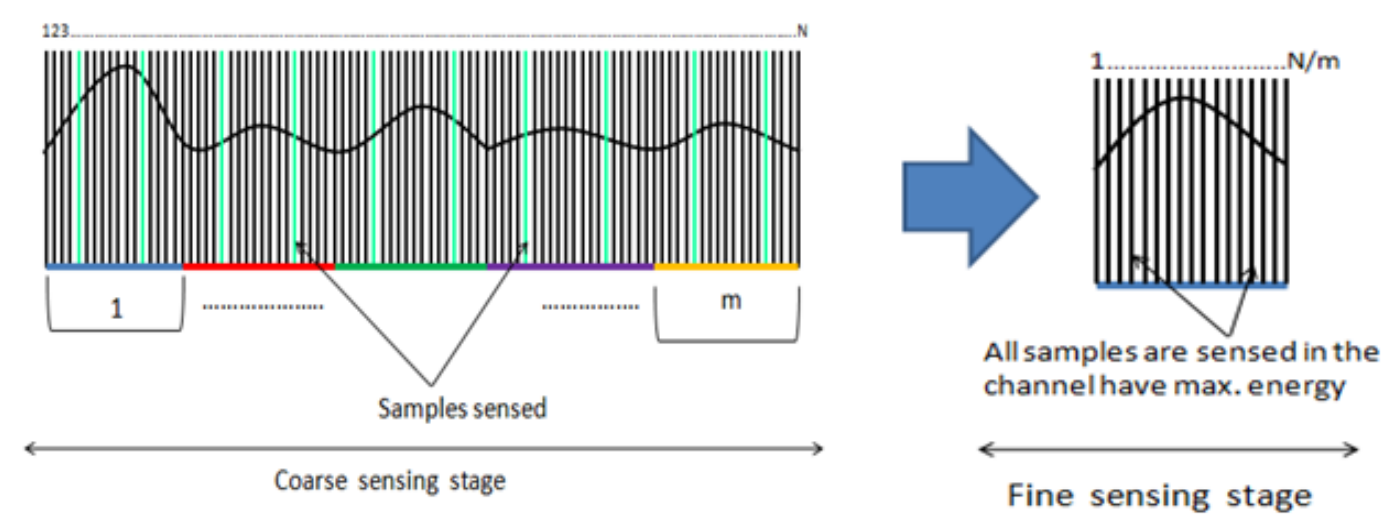

FIGURE 2. The division of spectrum using the proposed method.

\begin{tabular}{|c|c|}
\hline Parameter Name & Value \\
\hline Bit rate & $2 \mathrm{Mbps}$ \\
\hline Number of channels $m$ & 10 \\
\hline Modulation type & QPSK (PU signal) \\
\hline Probability of false alarm & $10^{-3}$ \\
\hline Sensing ratio $k$ & $16 \%$ \\
\hline \multicolumn{2}{|l|}{ Total number of samples } \\
\hline in the spectrum $N$ & 1000 \\
\hline Samples per symbol & 100 \\
\hline Distance $d$ & $10 \mathrm{~m}$ \\
\hline
\end{tabular}

TABLE 1. Simulation parameters.

$C_{\mathrm{ss}, j}=8 \mathrm{~nJ}$ will be used.

According to the proposed algorithm, (7) can be written as

$$
C_{\mathrm{s}, j}=C_{\mathrm{co}, j}+C_{\mathrm{fi}, j},
$$

where $C_{\mathrm{co}, j}$ and $C_{\mathrm{f}, j}$ are the energy consumptions in the coarse and fine stages respectively, with

$$
C_{\mathrm{co}, j}=N k C_{\mathrm{ss}, j}, \quad C_{\mathrm{fi}, j}=\frac{N}{m} C_{\mathrm{ss}, j},
$$

where $k$ is the sensing ratio, which is defined as the number of sensed samples to the total number of samples $N$, and $m$ is the number of channels in the spectrum. Figure 2 explains the division process of the spectrum used in the proposed coarse-fine sensing method.

So, the total energy consumption per sensor in both coarse and fine stages is:

$$
C_{\mathrm{s}, j}=C_{\mathrm{co}, j}+C_{\mathrm{fi}, j}=\left(k+\frac{1}{m}\right) N C_{\mathrm{ss}, j} .
$$

The amount of energy consumption per bit in the transmission stage is computed as follows: according to [15], the consumed energy related to the signal processing part in the transmission mode for a data rate of $250 \mathrm{~kb} / \mathrm{s}$, a voltage of $2.1 \mathrm{~V}$, and current of

\begin{tabular}{cccc}
\hline Tap & $\begin{array}{c}\text { Relative } \\
\text { delay (ns) }\end{array}$ & $\begin{array}{c}\text { Average } \\
\text { power }(\mathrm{dB})\end{array}$ & $\begin{array}{c}\text { Doppler } \\
\text { spectrum }\end{array}$ \\
\hline 1 & 0 & 0 & flat \\
2 & 50 & -3 & flat \\
3 & 110 & -10 & flat \\
4 & 170 & -18 & flat \\
5 & 290 & -26 & flat \\
6 & 310 & -32 & flat \\
\hline
\end{tabular}

TABLE 2. Multipath fading properties of ITU indoor channel model (A).

$17.4 \mathrm{~mA}$ is approximately $150 \mathrm{~nJ} /$ bit. The transmitter dissipates energy to run the radio electronics and the power amplifier. To transmit one bit over a distance $d$, the radio spends:

$$
C_{\mathrm{tb}, j}=C_{\mathrm{t}-\mathrm{elec}, j}+e_{\mathrm{amp}, j} d^{2},
$$

where $C_{\mathrm{t} \text {-elec, } j}$ is the transmitter electronics energy and $e_{\mathrm{amp}, j}$ is the amplification required to satisfy a given receiver sensitivity level. Assuming a data rate of $250 \mathrm{~kb} / \mathrm{s}$ and a transmission power of $20 \mathrm{~mW}$, $C_{\mathrm{t} \text {-elec }, j}=80 \mathrm{~nJ}$. To satisfy a receiver sensitivity of $90 \mathrm{dBm}$ at an SNR of $10 \mathrm{~dB}, e_{\mathrm{amp}, j}$ is $40.4 \mathrm{pJ} / \mathrm{m} 2$. So, the transmitted energy per bit for the $j$ th $\mathrm{CU}$ would be

$$
C_{\mathrm{t}, j}=C_{\mathrm{tb}, j}+150 \mathrm{~nJ} / \mathrm{bit} .
$$

Substituting (13) and (11) into 6 we get

$$
C_{j}=\left(k+\frac{1}{m}\right) N C_{\mathrm{ss}, j}+C_{\mathrm{tb}, j}+150 \mathrm{~nJ} / \mathrm{bit} .
$$

In this work, the OR rule is used in the FC to make the final decision. This way, the global probability of the detection $Q_{\mathrm{d}}$ is 11 .

$$
Q_{\mathrm{d}}=1-\prod_{j=1}^{M}\left(1-P_{\mathrm{d}}\right)
$$

where $P_{\mathrm{d}}$ is the local probability of the detection and $M$ is the number of cognitive users. 


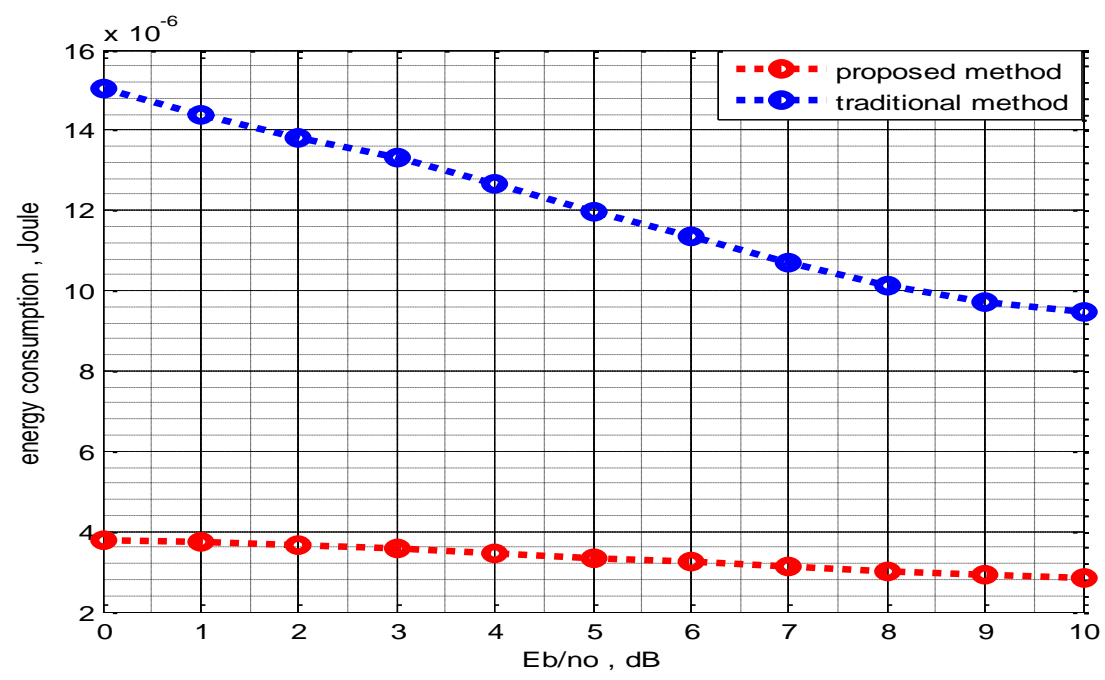

FiguRE 3. Energy consumption versus $E_{\mathrm{b}} / N_{\mathrm{o}}$ in AWGN channel.

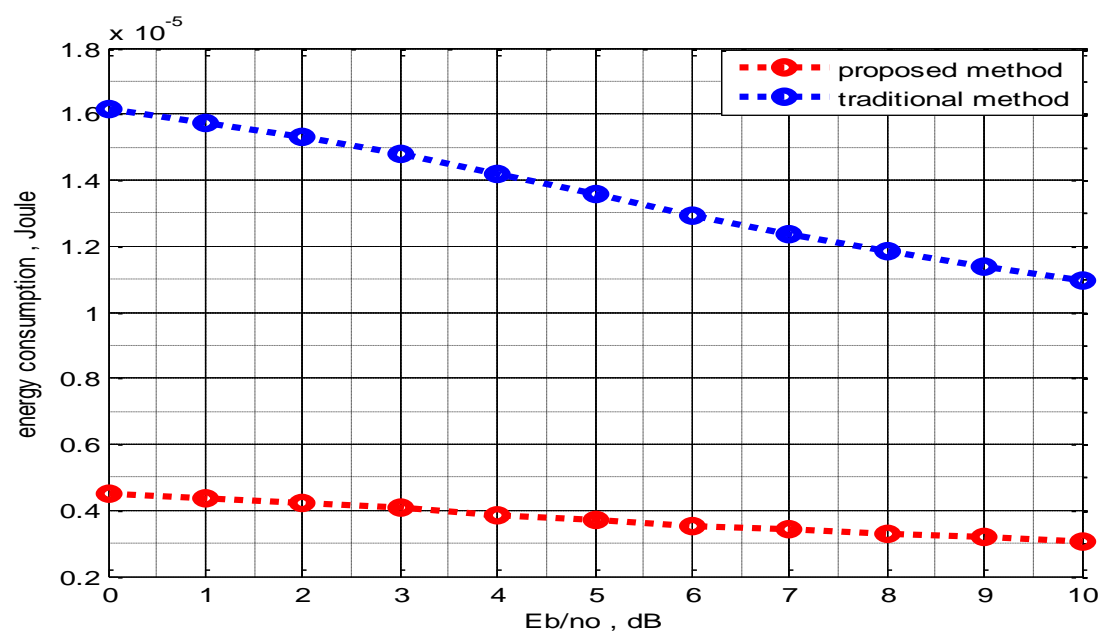

FiguRE 4. Energy consumption versus $E_{\mathrm{b}} / N_{\mathrm{o}}$ in Rayleigh multipath fading channel.

\section{Simulation Results AND DISCUSSION}

This section presents the simulation results of energy detector performance and the energy consumption of the proposed scheme under the cooperative and non-cooperative scenario. They are divided into two parts: the first part shows the results of the proposed method using IEEE 802.15.4/ZigBee standard as a case study in the CU. The performance is tested under the AWGN and Rayleigh multipath fading channels, all results in this part include the amount of the energy consumption of the $\mathrm{CU}$ in the sensing and transmission stages. To evaluate the performance of the proposed scheme, it is compared with a traditional sensing method, which senses all spectrum samples (i.e $\mathrm{k}=1$ ). The second part shows the performance comparison between the proposed method and the censoring and sequential censoring used in [11.

\subsection{PART ONE}

The simulation parameters used in this part are shown in Table 1 The used multipath fading is "ITU indoor channel model A" as shown in Table 2 16.

\subsubsection{NON-COOPERATIVE SCENARIO}

Figures 3 and 4 show the performance curves of the energy consumption of the CU versus $E_{\mathrm{b}} / N_{\mathrm{o}}$ in the AWGN and Rayleigh multipath fading channels respectively to the proposed method as compared with the traditional method. In Figure 3 , it can be seen that the energy consumption reduces as $E_{\mathrm{b}} / N_{\mathrm{o}}$ increases, since a fewer number of sensing samples is required by the $\mathrm{CU}$ in the sensing process to detect the PU signal when $E_{\mathrm{b}} / N_{\mathrm{o}}$ is high. It is also noted that the proposed algorithm achieves an improvement in the energy consumption when compared with the traditional method. For example, when $E_{\mathrm{b}} / N_{\mathrm{o}}$ equals $5 \mathrm{~dB}$, energy consumption decreases by $55 \%$ in the 


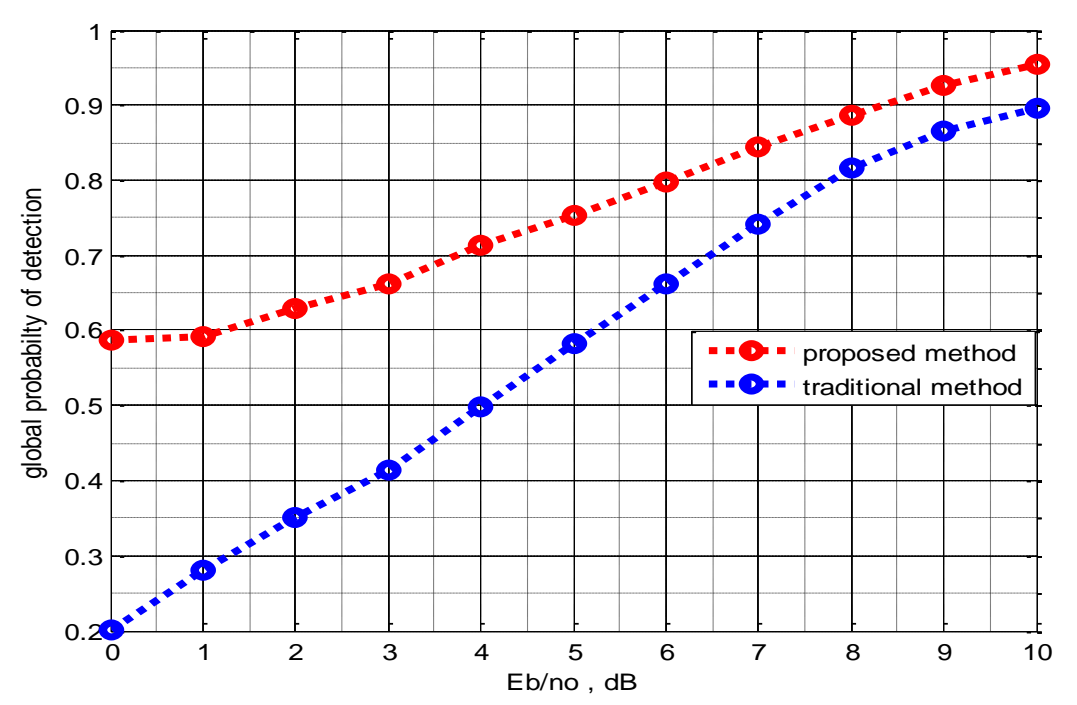

Figure 5. Pd versus $E_{\mathrm{b}} / N_{\mathrm{o}}$ in AWGN channel.

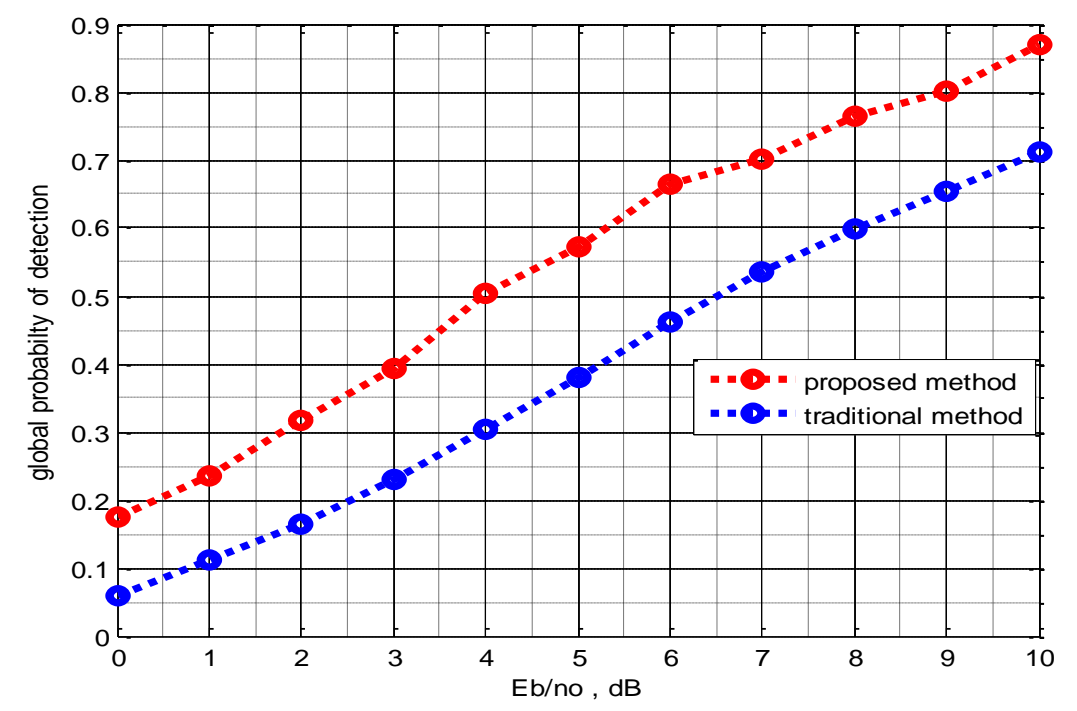

Figure 6. Pd versus $E_{\mathrm{b}} / N_{\mathrm{o}}$ in Rayleigh multipath fading channel.

proposed method. Figure 4 shows the same performance in Figure 3, but with an increase in energy consumption due to the effect of the multipath fading. Figures 5 and 6 show the performance curves of probability of detection versus $E_{\mathrm{b}} / N_{\mathrm{o}}$ in the AWGN and Rayleigh multipath fading channels respectively. It can be seen that in Figure 5, the detection performance increases as $E_{\mathrm{b}} / N_{\mathrm{o}}$ increases and the proposed method outperforms the traditional method. It can be seen that the improvement of the probability of detection in the proposed method becomes more significant in the small values of $E_{\mathrm{b}} / N_{\mathrm{o}}$. For example, when $E_{\mathrm{b}} / N_{\mathrm{o}}$ equals $3 \mathrm{~dB}, \mathrm{Pd}$ is increased from 0.42 in traditional method, to 0.7 in the proposed method. Figure 6 shows the same performance as in Figure 5. but with a slight reduction in detection performance due to the effect of the multipath fading. The reason behind the improvement in the detection performance in the proposed method is the use of the fine sensing stage, which senses the channel with a sensing ratio of $100 \%$ and that increases the accuracy in the performance detection.

\subsubsection{CoOperative SCEnARIO}

Figures 7 and 8 show the performance curves of energy consumption and $Q_{\mathrm{d}}$ versus $E_{\mathrm{b}} / N_{\mathrm{o}}$ respectively. In the cooperative scenario, $5 \mathrm{CUs}$, and the OR rule in the FC for the final decision are used. The scenarios considered assume that only $3 \mathrm{CUs}$ out of $5 \mathrm{CUs}$ are suffering from multipath fading. 


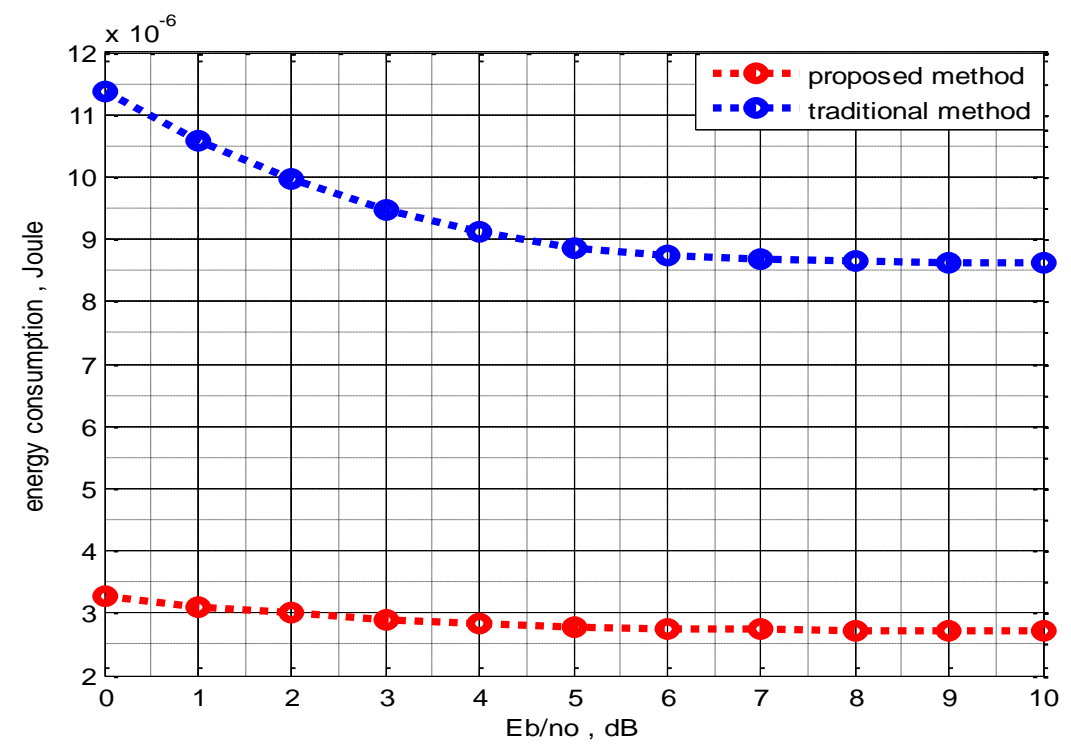

Figure 7 . Energy consumption versus $E_{\mathrm{b}} / N_{\mathrm{o}}$ using 5 CUs.

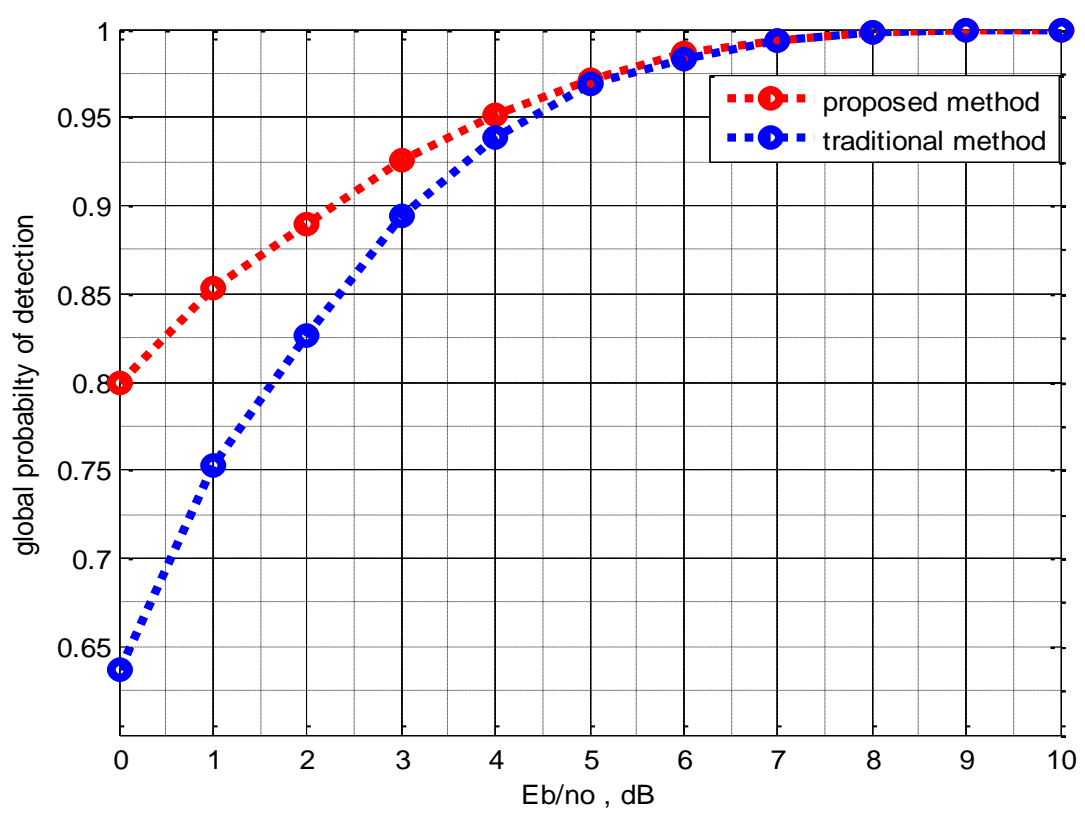

Figure $8 . Q_{\mathrm{D}}$ versus $E_{\mathrm{b}} / N_{\mathrm{o}}$ using 5 CUs.

As shown in Figure 7, the average energy consumption per sensor is increased as $E_{\mathrm{b}} / N_{\mathrm{o}}$ increases. When we make a comparison of this figure with a non-cooperative scenario given in Figure 4 it can be seen that the reduction in the energy consumption is increased in both traditional and proposed methods. For example, when $E_{\mathrm{b}} / N_{\mathrm{o}}$ equals $5 \mathrm{~dB}$, the energy consumption reduces by $60 \%$ in the proposed method. The reason behind this improvement is that the CUs will share the statistics about the PU existence, which will increase the overall detection probability as shown in Figure 8 Also, in Figure 8, it can be seen that the proposed method has a significant improvement in detection performance when compared to the tradi- tional method. This is because of the use of two stages of the detection process, which increase performance detection, especially in fine sensing.

\subsection{PART TWO}

In this section, the performances of the proposed method and censoring and sequential censoring methods discussed in 11 are evaluated and compared. In these results, we use the same parameters that are used in both methods. The parameters are $M=5$, $N=30, C_{\mathrm{ss}, j}=1, C_{\mathrm{t}, j}=10, \alpha=0.1, \beta=0.9$, where $\alpha$ is the probability of a false alarm constraint , $\beta$ is the probability of detection constraint. As stated in [11, $Q_{\mathrm{D}} \geq \beta$. The FC uses the OR rule and there 


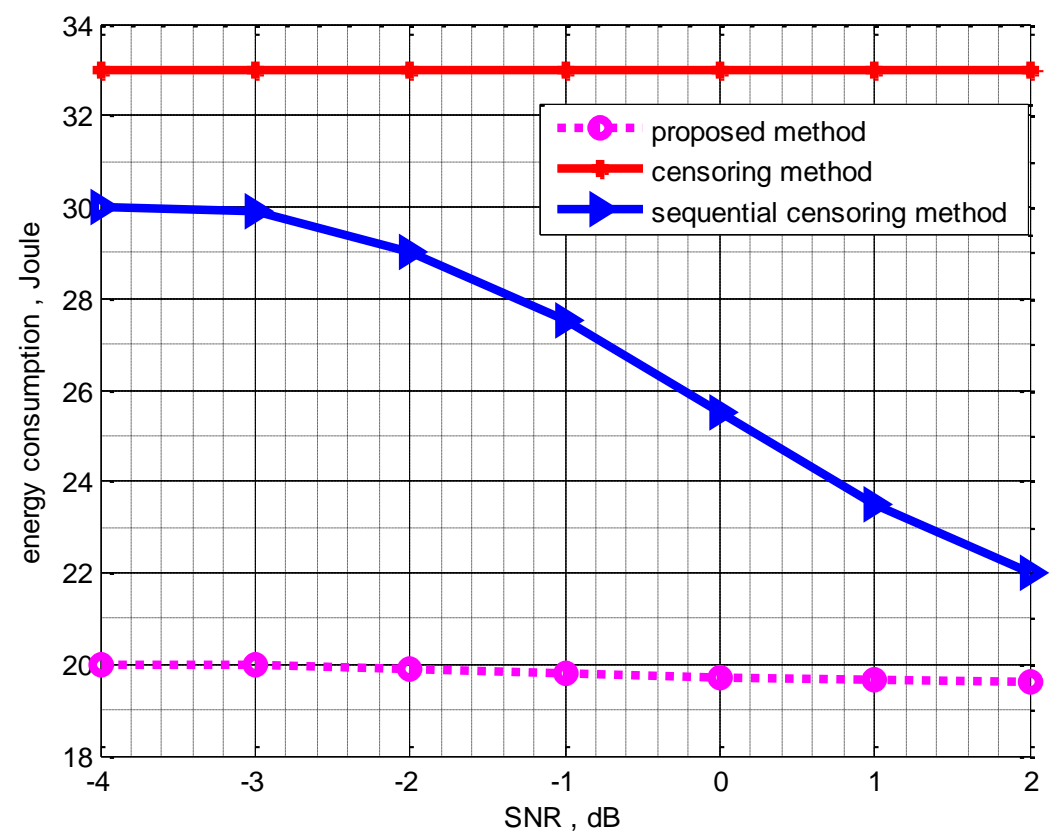

FiguRE 9. Average energy consumption per sensor vs. SNR, $\pi_{0}=0.2$.

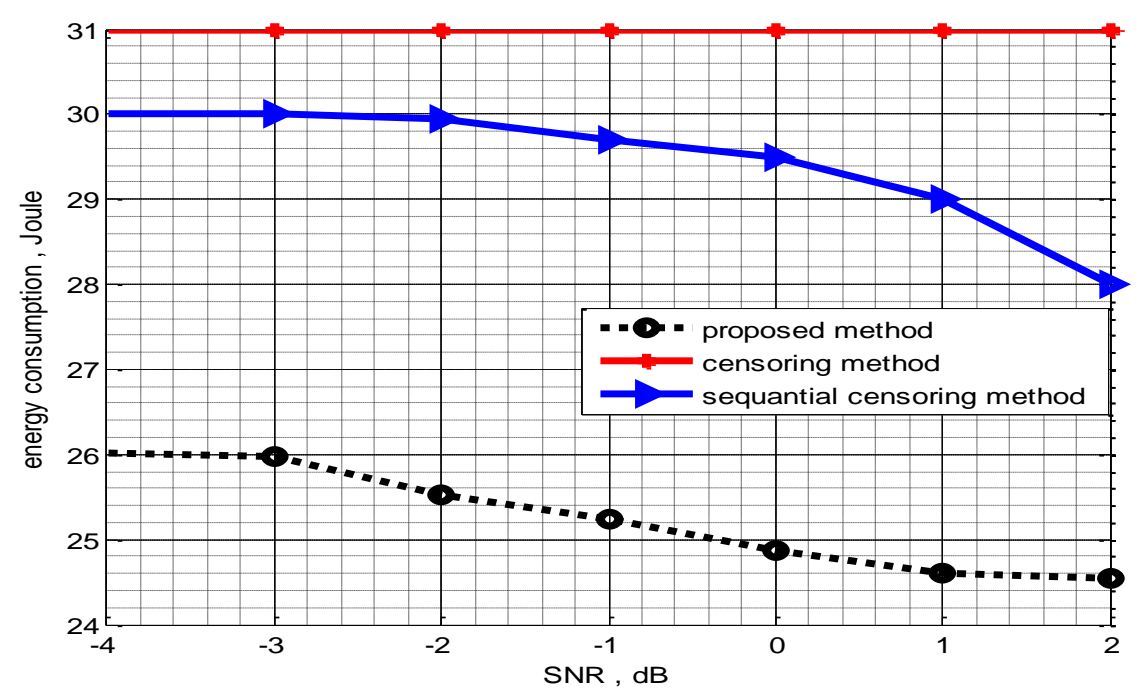

FIgURE 10. Average energy consumption per sensor vs. SNR, $\pi_{0}=0.8$.

are predefined values for initial probabilities of the PU absence $\left(\pi_{0}\right)$ and presence $\left(\pi_{1}\right)$ [11]. When adding this probabilities to the spectrum sensing problem, the new expression for the energy consumption by $j$ th CU will be $C_{\mathrm{s}, j}=\pi_{0} C_{\mathrm{co}, j}+\pi_{1} C_{\mathrm{fi}, j}$. It is worth noted that when $\pi_{0}$ is 0 or 1 , the CU enters into a sleep mode and the sensing process is stopped. Figures 9 and 10 show the performance curves of an average energy consumption per sensor versus the SNR when $\pi_{0}$ equals 0.2 and 0.8 respectively. In these two figures, it can be seen that the proposed method improves the energy consumption, as censoring and sequential censoring methods and this improvement increase as $E_{\mathrm{b}} / N_{\mathrm{o}}$ decreases. For example, in Figure 9 at $\mathrm{SNR}=0 \mathrm{~dB}$, the energy consumption is reduced by $45 \%, 35 \%$ when we compare it with the censoring, sequential censoring methods, respectively.

We also note that in the proposed method, the energy consumption stays at a constant level as the SNR increases. This can be interpreted as follows: when the SNR increases the detection probability increases and reaches its full scale value at a higher SNR value, keeping the performance at constant level, which, as a result, makes the energy consumption constant. Figure 11 shows the performance curve of the global probability of the detection versus the SNR of the proposed system. This figure shows that the proposed system operates in the right range to 


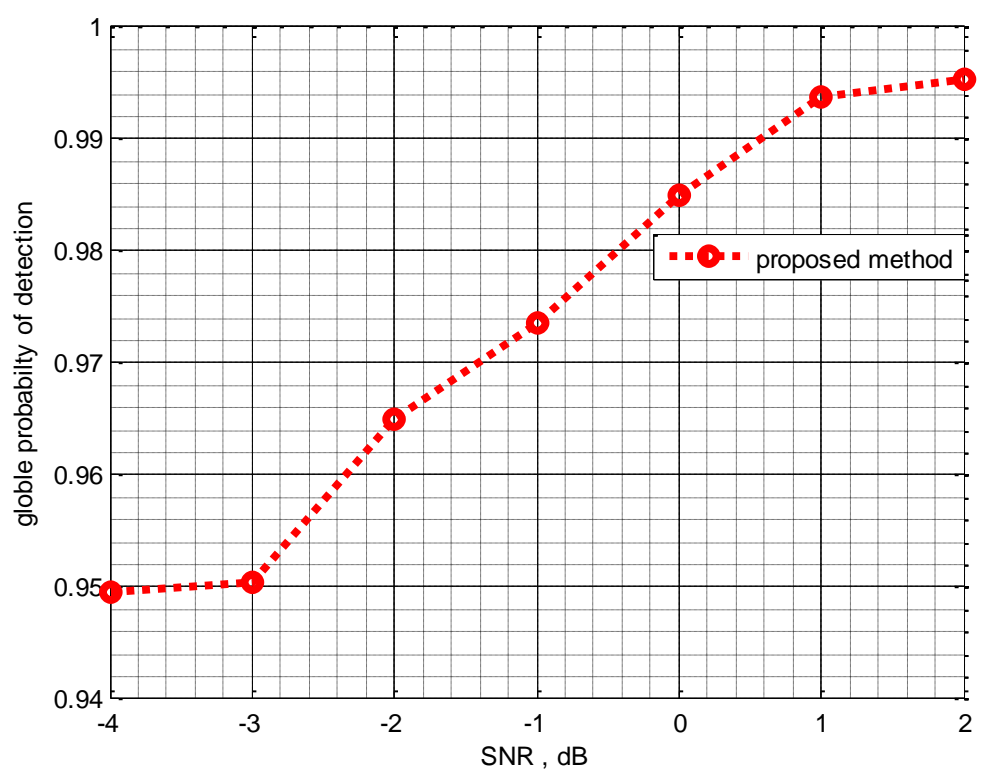

FiguRE 11. Global probability of detection versus SNR.

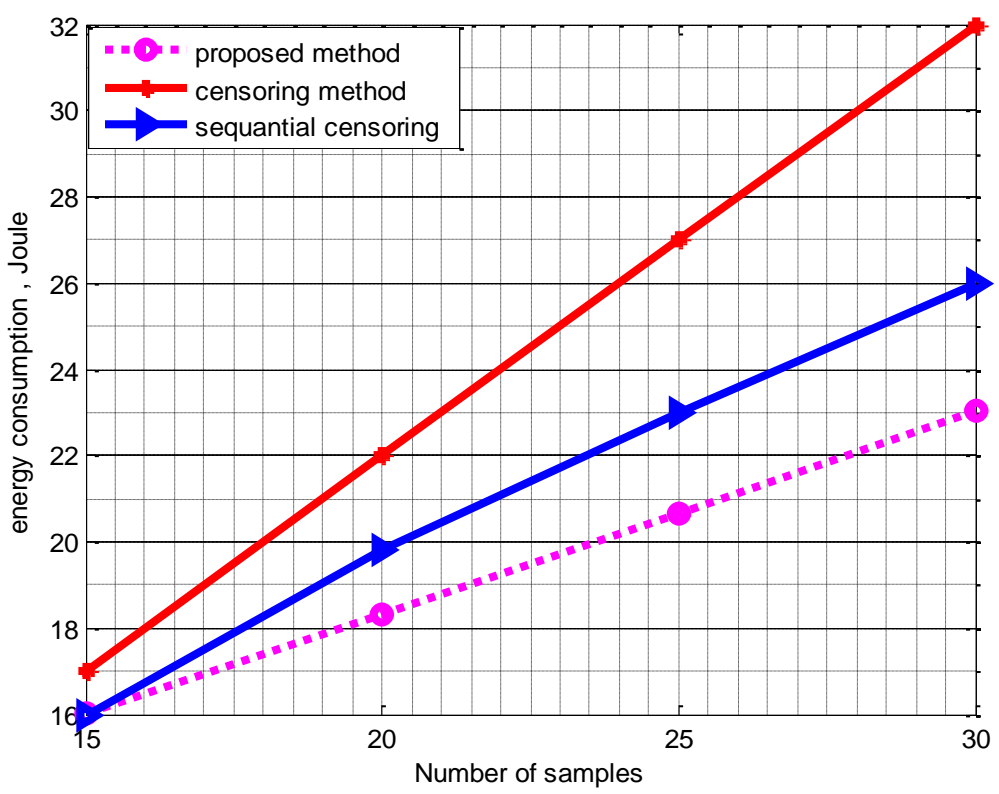

Figure 12. Average energy consumption per sensor vs. no of samples, $\pi_{0}=0.5$.

make a fair performance comparison with the method in [11, since the relation $Q_{\mathrm{D}} \geq \beta$ is satisfied $(\beta=0.9$ in simulation parameters). Figure 12 shows the performance curve of the average energy consumption per sensor versus the number of samples. The parameter used in this figure is the same as used in Figures 9 and 10 , but in this results, $\pi_{0}$ is 0.5 , and the SNR is a vector of $[1 \mathrm{~dB}, 2 \mathrm{~dB}, 3 \mathrm{~dB}, 4 \mathrm{~dB}, 5 \mathrm{~dB}]$ for 5 CUs. In this figure, it can be seen that on the one hand, the energy consumption is increased when the number of samples is increased, but on the other hand, the proposed method has a significant improvement in the energy consumption, especially for a high number of sensed samples. This is because the high number of samples will increase the performance of the detection and this leads to the decrease in the energy consumption.

\section{Conclusions}

In this research, we proposed an efficient method to reduce the energy consumption with improvement in performance detection in the $\mathrm{CR}$ networks. The simulation results are shown as comparisons with traditional method in two scenarios: cooperative and non-cooperative, and with censoring and sequential censoring methods. The results have shown a sig- 
nificant improvement in the energy consumption by the proposed method. The main reason behind this improvement is that the proposed algorithm combines both improving in the energy consumption by reducing the number of sensed samples and increase in detection probability. The improvement in energy consumption is caused by the fixed length coarse sensing stage by using fewer number of samples, while the increase in the detection performance is caused by the use of the fine sensing stage. The impact of the proposed scheme becomes more clear at a low signalto-noise ratio values and in the presence of a high initial probability about the primary user availability.

\section{ACKNOWLEDGEMENTS}

The authors would like to thank the head of Networks Engineering departement at Al-Nahrain university - college of information engineering for providing the facilities and support to carry out this research.

\section{REFERENCES}

[1] Sheetal Kokare and R.D.Kamble, "Spectrum Sensing Techniques in Cognitive Radio Cycle," International Journal of Engineering Trends and Technology (IJETT), Vol. 9, No. 1, March 2014.

[2] Saud Althunibat et al., "Software-Defined and Cognitive Radio Technologies for Dynamic Spectrum Management," United States of America, DOI: 10.4018/978-1-4666-6571-2.ch004, MARCH 2015

[3] M. Emara et al.," Spectrum Sensing Optimization and Performance Enhancement of Cognitive Radio Networks" , Wireless Personal Communications, DOI 10.1007/s11277-015-2962-5, 2015.

[4] Saud Althunibat "Towards Energy Efficient Cooperative Spectrum Sensing in Cognitive Radio Networks ", Ph.D thesis, University of Trento, November 2014.

[5] S. J. Kim, G. Li, and G. B. Giannakis, "Multiband Cognitive Radio Spectrum Sensing for Real-Time Traffic", IEEE Transactions on Signal Processing, May 2010 .

[6] S. J. Kim, and G. B. Giannakis, "Sequential and Cooperative Sensing for Multi-Channel Cognitive Radios," IEEE Transactions on Signal Processing, vol.58, no.8,pp.4239-4253, August. 2010.
[7] S. J. Kim and G. B. Giannakis, "Rate-Optimal and Reduced-Complexity Sequential Sensing Algorithms for Cognitive OFDM Radios", EURASIP Journal on Advances in Signal Processing, vol. 2009, Article ID 421540, 2009.

[8] Q. Zou, S. Zheng and A. H. Sayed, "Cooperative Sensing via Sequential Detection," IEEE Transactions on Signal Processing, vol.58, no.12, pp.6266-6283, December 2010.

[9] Y. Xin and H. Zhang, "A Simple Sequential Spectrum Sensing Scheme for Cognitive Radios," submitted to IEEE Transactions on Signal Processing, available on http://arxiv.org/PS cache/arxiv/pdf/0905/0905.4684v1.pdf.

[10] P. Cheng et al., "Energy-efficient cooperative spectrum sensing in sensor aided cognitive radio networks" ,IEEE Wireless Communications, vol. 19, no. 6, 2012, pp. 100-105.

[11] S. Maleki and G. Leus, "Censored Truncated Sequential Spectrum Sensing for Cognitive Radio Networks" , IEEE Journal on Selected Areas in Communications, vol. 31, no. 3, March 2013.

[12] O. Ergul, and O. Akan ,"Cooperative Coarse Spectrum Sensing for Cognitive Radio Sensor Networks", IEEE WCNC' in track2 (MAC and cross-layer Design), 2014.

[13] Hikmat N. Abdullah and Hadeel S. Abed, "Improvement of Energy Consumption in Cognitive Radio by Reducing the Number of Sensed Samples", proceeding of IEEE Al-Sadiq International conference on Multidisciplinary in IT and Communication Science and Technologies (AIC-MITC), 9-10 May 2016, Baghdad, Iraq, pp. 301-306.

[14] Hikmat N. Abdullah and Hadeel S. Abed, "Energy Consumption Control in Cooperative and Noncooperative Cognitive Radio using Variable Spectrum Sensing Sampling", Journal of Telecommunication, Electronic and Computer Engineering (JTEC), Vol- 8, Issue- 9, pp. 7-12, Malaysia, December 2016.

[15] S. Maleki, A Pandharipande, and G. Leus , "Energy-Efficient Distributed Spectrum Sensing for Cognitive Sensor Networks", IEEE Sensor Journal, vol.11, no. 3, March 2011.

[16] M. Pätzold, "Mobile Fading Channels", John Wiley \& Sons, Ltd, first edition, 2000 